\title{
The effect of dietary carbohydrates and polyunsaturated fatty acids on red blood cell membrane lipid profile in a cohort of cancer patients
}

\author{
I. Tueros ${ }^{1}$, J. Amézaga ${ }^{1}$, F. Roux ${ }^{1}$, S. Arranz ${ }^{1}$ A. Larraioz ${ }^{2}$, G. Ugartemendia $^{2}$, \\ A. Urruticoechea ${ }^{2}$, C. Ferreri ${ }^{3}$ and M. Uriarte \\ ${ }^{1}$ AZTI, Food and Health, Derio, Spain, \\ ${ }^{2}$ Fundación Onkologikoa, Donostia, Spain and \\ ${ }^{3}$ ISOF-CNR, Bologna, Italy.
}

Epidemiological or intervention studies relating to dietary factors, can be challenging to interpret because of food and eating patterns complexity. Data driven 'omics' may contribute to more rapid advancement in our understanding of diet and cancer and their interacting metabolic pathways. Among them, membrane lipidomics is related to cell membrane structure and results particularly interesting as it determines the contribution of exogenous factors (such as lipids and carbohydrates from diet) to the cell membrane structure which is determinant in cell function, allowing as well, in-depth study of human metabolism[1]. The objective of this work is to study the contribution of dietary nutrients (carbohydrates, sugars and lipids) to red blood cell membrane (RBC) lipid profile and the connection with metabolic signatures in cancer patients.

A prospective observational study was carried out on a cohort of adult cancer patients undergoing chemotherapy at the Oncology Outpatient Unit from Onkologikoa Foundation. Dietary habits and chemosensory alterations were recorded in a validated food frequency questionnaire that patients completed via personal interviews. Fatty acid-based lipidomic profiles were obtained evaluating the fatty acid composition of erythrocyte membrane phospholipids by GC-MS in cancer patients $(n=63)$ and in a control group of general healthy population $(n=43)[2]$.

Regarding correlations between dietary nutrients and RBC lipid profile, the intake of saturated fats did not correlate with saturated fatty acids in RBC membrane. However, total carbohydrate intake correlated with $\mathrm{C} 18: 0(0.26 ; \mathrm{p}=0.013)$, and refined sugars did correlate with $\mathrm{C} 16: 0(\mathrm{r}=0.24 ; \mathrm{p}=0.024)$ and $\mathrm{C} 18: 0(\mathrm{r}=0.59 ; \mathrm{p}=0.000)$ levels in $\mathrm{RBC}$ membrane. This could be connected to de novo lipogenesis pathway that is known to be activated in cancer disease [3]. On the other hand, the polyunsaturated fatty acid EPA correlated with the corresponding RBC EPA $(r=0.27 ; p=0.009)$. This could be connected to essentiality of this dietary lipid. In the adjusted model, lipidomic profiles revealed higher levels of omega 6 (n-6 C18:2, 15.7 \% and n6-C20:3, 22.2 \%) and $\Delta 9$ desaturase activity $(28.3 \%)$ in cancer patients compared to the general healthy population. These unbalances were not related to dietary habits. These metabolic features are connected to inflammation and lipid biosynthesis respectively, which are hallmarks of cancer metabolism.

In conclusion, RBC membrane lipidomics resulted in a comprehensive tool for the study of diet and metabolism. It can provide new insights not only for the study of metabolic changes occurred in cancer patients, but also for the development of nutritional recommendations for this population.

1. Tueros I.; Uriarte M. Innovative food products for cancer patients: future directions. J Sci Food Agric 2018, 98, 1647-1652.

2. Sansone A.; Tolika E.; Louka M.; Sunda V.; Deplano S.; Melchiorre M.; Anagnostopoulos D.; Chatgilialoglu C.; Formisano C.; Di Micco R., et al. Hexadecenoic Fatty Acid Isomers in Human Blood Lipids and Their Relevance for the Interpretation of Lipidomic Profiles. PLoS ONE 2016, 11.

3. Igal R.A. Stearoyl CoA desaturase-1: New insights into a central regulator of cancer metabolism. Biochim Biophys Acta $2016,12,14$. 